EPJ Web of Conferences 59, 08006 (2013)

DOI: $10.1051 /$ epjconf/20135908006

(C) Owned by the authors, published by EDP Sciences, 2013

\title{
Thermal modelling of the calorimeter used for energy measurement of LMJ laser pulse
}

\author{
C. Crespy ${ }^{\mathrm{a}}$ and D. Villate
}

Commissariat à l'Énergie Atomique et aux Énergie Alternatives DAM, CESTA, BP. 2, 33114 Le Barp, France

\begin{abstract}
In this work, a 3D thermal model of the LMJ calorimeter is developed using Comsol multiphysics. The unknown thermal properties of the system are identified by comparing the model results with the measured temperature. Several model's applications, such as comparing deposition modes (electrical and optical) or defining calibration procedure, are presented.
\end{abstract}

\section{INTRODUCTION}

The Laser Integration Line (LIL) at Commissariat à l'Energie Atomique (CEA) near Bordeaux (France) is a prototype beam line, built to demonstrate the technology and performance of the French Laser Mégajoule (LMJ) ignition facility [1]. Electrically calibrated laser calorimeters are considered as the most suitable tools to perform laser energy measurements. REC calorimeter has been especially manufactured to accurately quantify the energy of laser pulses of the LIL. REC consists of an absorbing glass which is attached to an aluminium plate. The response of the calorimeter is the time dependent temperature profile measured on the rear face of the aluminium plate using an array of Peltier cells. Moreover, electrical heaters are attached to the aluminium rear face to perform electrical calibration. (cf. figure 1).

The energy deposited inside the calorimeter is dissipated into the thermal mass. Obviously, modelling and analysis of the calorimeter thermal behaviour are very important issues. In this study, a three-dimensional model is developed with COMSOL Multiphysics to analyze the calorimeter response.

\section{THERMAL MODEL}

Since the radiative emission can be neglected inside the glass, an exponential decay function is used to model the laser pulse absorption. Thus, the combined radiative-conductive transfers inside the glass are written (cf. [2, 3]):

$$
\begin{gathered}
\rho_{v} C_{P, v} \frac{\partial T(x, y, z, t)}{\partial t}=-\nabla q^{c}-\nabla q^{r} \\
\text { with : } q^{c}=-k_{v} \cdot \overrightarrow{g r a d} T \text { and } q^{r}(x, t)=\left(1-R_{f}\right) \frac{Q e^{-\beta x}}{\tau d o^{2}}
\end{gathered}
$$

\footnotetext{
ae-mail: charles.crespy@cea.fr
}

This is an Open Access article distributed under the terms of the Creative Commons Attribution License 2.0, which permits unrestricted use, distribution, and reproduction in any medium, provided the original work is properly cited. 

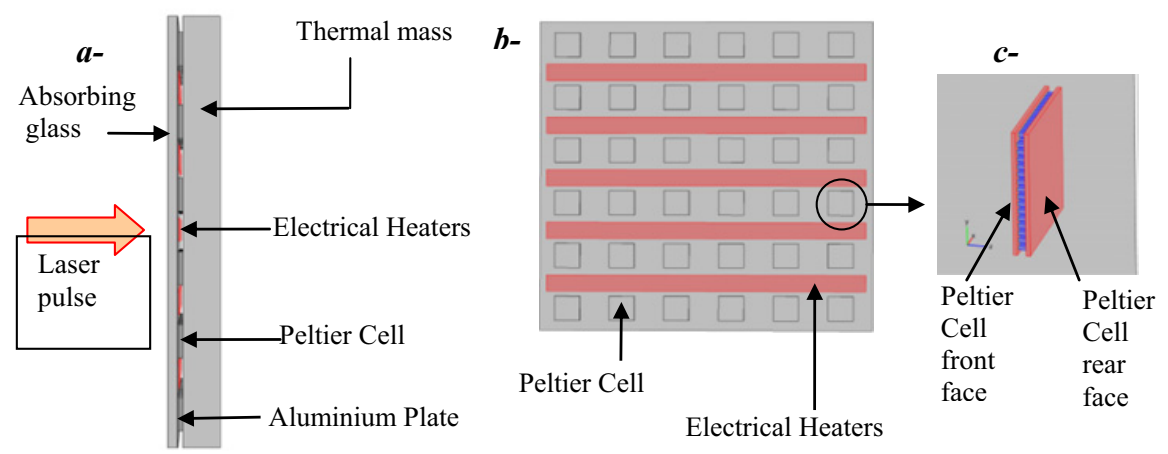

Figure 1. REC calorimeter (a-profile view, b- rear face of the aluminium plate, c- detail: Peltier Cell).
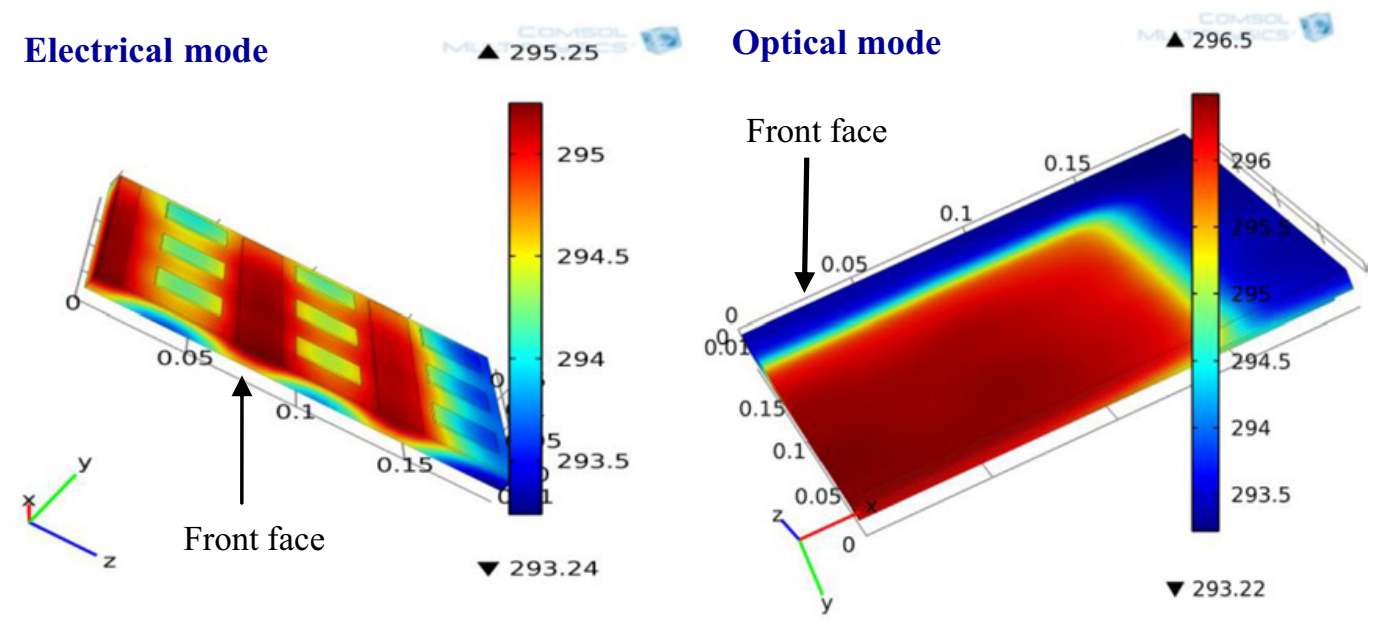

Figure 2. Visualization of the temperature fields for the two deposition mode (because of the symmetry only a quarter of the calorimeter is modelled).

$Q$ is the pulse energy, $R_{f}$ : reflectivity of glass, $\tau$ pulse duration, $d_{0}$ laser beam size, $\rho_{v} C p$ and $k_{v}$ are respectively the density, the specific heat and the conductivity of the glass. The pulse duration in optical mode is close to one second, while it's close to 15 seconds in electrical mode.

Concerning the thermal exchange with the environment, the radiative, convective and conductive phenomenons are modelled. It should be noted that the radiative transfer between aluminium plate and thermal mass is also considered. To reduce the computation time all the semi conductive couples inside a Peltier cell are modelled using a homogenous virtual material. Figure 2 shows the temperature fields inside the glass and the aluminium plate for the two deposition modes.

The calorimeter response is the temperature difference between front and rear faces of the Peltier cells integrated over the 36 Cells (cf. fig. 3). It should be noted that when the response of the calorimeter is close to zero, the temperature of the system is uniform but not equal to the ambient temperature. The amplitude signal is used to estimate the amount of energy deposited inside the calorimeter. The unknown thermal properties of the system (such as thermal properties of Peltier Cells and thermal resistance due to the paste) are deduced by comparing the model results with the measured temperature profiles. Once the model calibration is performed, the maximum difference between simulated and measured profiles is less than $3 \%$ for the two deposition modes. The model error on amplitude signal is close to $0.5 \%$. The measured response and the simulated one are compared on figure 3. 

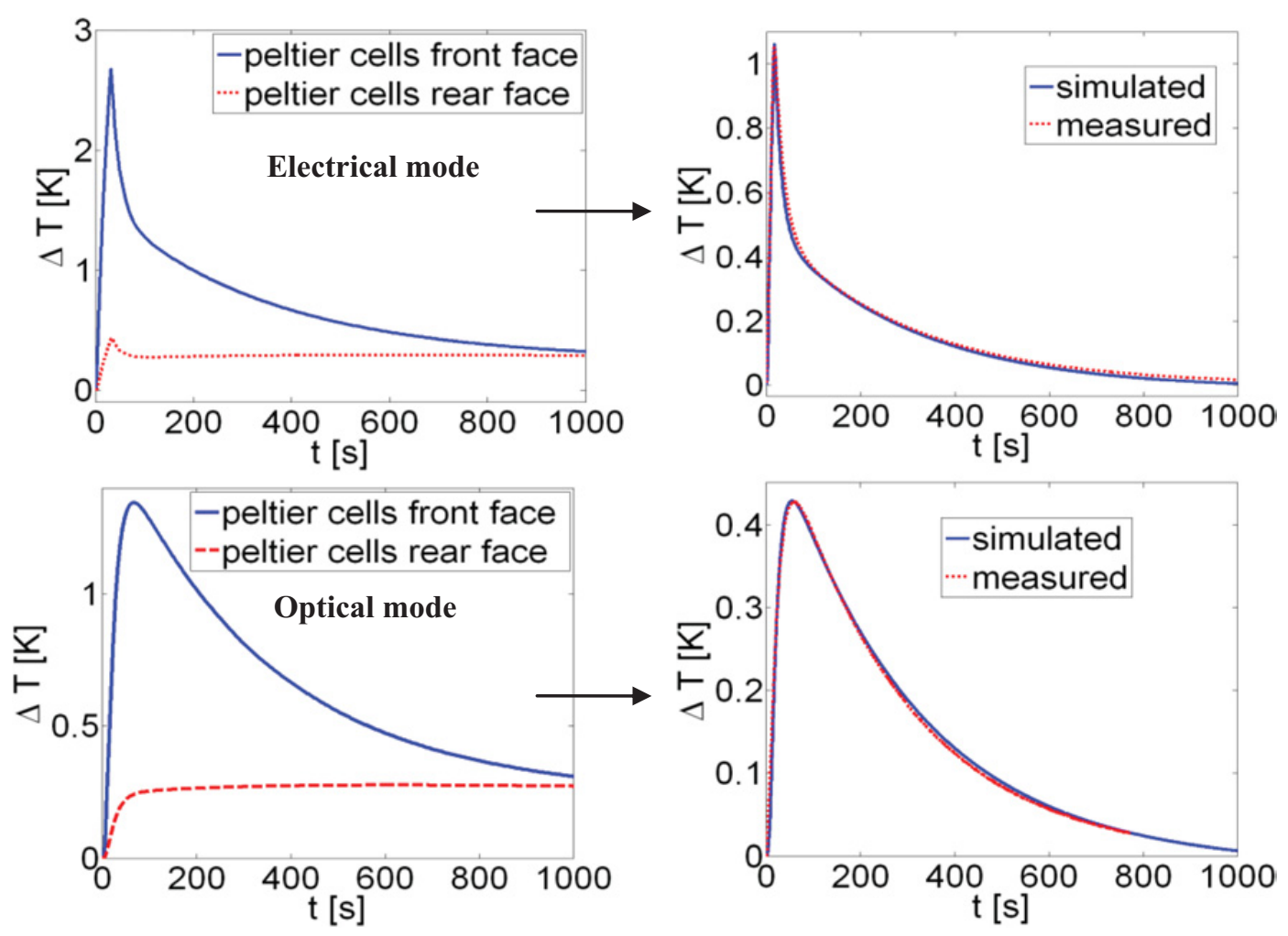

Figure 3. Calorimeter response simulation for the two deposit modes.

\section{RESULTS AND ANALYSIS}

\subsection{Energy balance}

The model is used to estimate the thermal behaviour difference between the two energy deposition modes. Thus, the fraction of energy dissipated in the environment (thermal losses) is estimated for both modes. In the optical mode, thermal losses are close to $20 \%$, when they are close to $17 \%$ in the electrical mode. This point is due to the difference of energy source location. In the optical mode, the energy is supplied on the front face. Thus, the time taken by the energy to reach the Peltier cells is longer than in electrical mode. During this time delay, a noticeable fraction of the energy is transmitted into the environment. Numerical results point out the non equivalence between the two deposition modes. Indeed, the thermal losses are too different to use electrical calibration to quantify laser energy. Thus, an optical calibration is required in order to perform accurate laser energy measurement.

\subsection{Calibration procedure optimisation}

$1000 \mathrm{~s}$ after an optical pulse, the measured signal is close to 0. However, the temperature inside the system is different from the ambient. The 3D model is used to quantify the influence of consecutive optical pulses on the calorimeter response. Consecutive pulses lead to a systematic error on the amplitude signal which can reach $3 \%$. The error decreases if the time between two consecutive pulses increases. Thus, for an accurate calibration, it's a requirement that the time between pulses is larger than $1500 \mathrm{~s}$ (error on signal amplitude less than $0.1 \%$ ). 

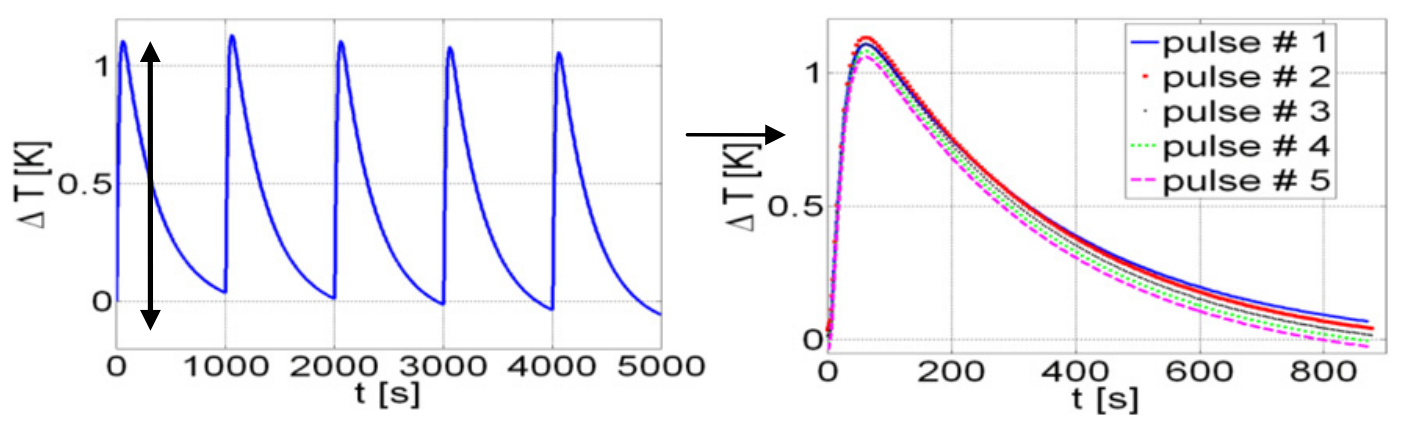

Figure 4. Simulation of the calorimeter response for consecutives laser pulses.
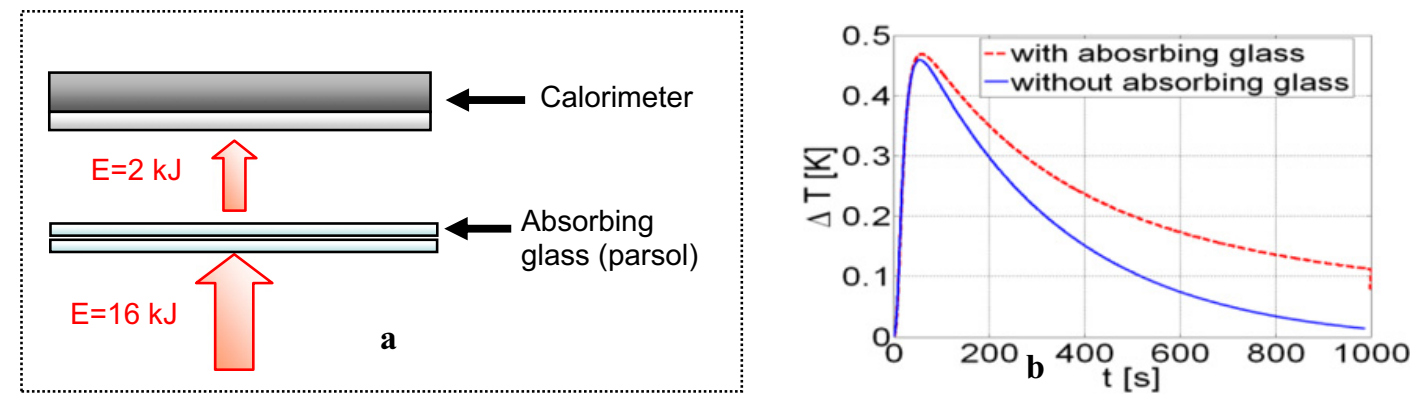

Figure 5. a) Experimental configuration for high energy pulse measurements. b) Influence of parsol on the calorimeter response.

\subsection{Measurement of high energy laser pulse}

In order to perform high energy laser pulses measurements $(16 \mathrm{~kJ})$, two slices of absorbing glass (parsol) are placed in front of the calorimeter. In this configuration, the amount of energy which reaches the calorimeter is close to $2 \mathrm{~kJ}$. However, the presence of hot absorbing glass in front of the calorimeter enhances the thermal exchanges between the calorimeter front face and the environment. The 3D model is used to quantify the influence of the absorbing glass slices on the calorimeter response.

The presence of parsol slices in front of the calorimeter leads to a change on the amplitude signal close to $2 \%$. This point is linked to the radiation flux emitted by the parsol slices on the front face of the calorimeter.

\section{CONCLUSION}

This study shows that the three dimensional model is a convenient tool to understand the heat transfer process within the REC calorimeter. In further works, all the unknown parameters will be measured and experimental devices will be implemented in order to validate the model. Thus, the model will be used to quantify environment influence on the calorimeter response. It is a first step to implement a post processing procedure to reduce the uncertainty on energy measurement of $L M J$ laser pulses.

\section{References}

[1] M. Di Nicola et al, Inertial Fusion Sciences and Applications, 2003, 558 (2004)

[2] D. H. Chen, Z.M. Zhang Int. J. of Heat and Mass Transfer 43, pp. 3061-3072 (2000)

[3] L. Liu, H. Tan, Int. J. Therm. Sci, 40, pp. 877-889 (2001) 